EPJ Web of Conferences 92,02026 (2015)

DOI: $10.1051 /$ epjconf/ 20159202026

(C) Owned by the authors, published by EDP Sciences, 2015

\title{
Modal Decomposition of Synthetic Jet Flow Based on CFD Computation
}

Tomáš Hyhlík ${ }^{1, a}$ and Hana Netřebská ${ }^{1}$

${ }^{1}$ CTU in Prague, FME, Department of Fluid Dynamics and Thermodynamics, Technická 4, 16607 Prague

\begin{abstract}
The article analyzes results of numerical simulation of synthetic jet flow using modal decomposition. The analyzes are based on the numerical simulation of axisymmetric unsteady laminar flow obtained using ANSYS Fluent CFD code. Three typical laminar regimes are compared from the point of view of modal decomposition. The first regime is without synthetic jet creation with Reynolds number $R e=76$ and Stokes number $\mathrm{S}=19.7$. The second studied regime is defined by $\mathrm{Re}=145$ and $\mathrm{S}=19.7$. The third regime of synthetic jet work is regime with $\mathrm{Re}=329$ and $\mathrm{S}=19.7$. Modal decomposition of obtained flow fields is done using proper orthogonal decomposition (POD) where energetically most important modes are identified. The structure of POD modes is discussed together with classical approach based on phase averaged velocities.
\end{abstract}

\section{Introduction}

Synthetic jet is zero net mass flux device, it means that there is no mass flux to its surroundings. There is only momentum transfer to the surroundings of synthetic jet. The possibility to generate (synthetize) jet is connected with irreversibility. Positive entropy production is leading to vorticity flux from the orifice in the blowing part of the oscillation period. If there is enough vorticity flux then vortex ring can be observed in the flow field. The created vortex ring is inducing velocity in the direction outside from the orifice. Synthetic jet can be generated only in the case where vortex ring induced velocity is able to overcome suction velocity. Phase averaged streamline velocity magnitude at jet centerline depicted in the figure 1 illustrates the case where synthetic jet is not created unlike the distribution in the figure 2 , where relatively weak synthetic jet is created.

\section{Parameters of Synthetic Jets}

This section is describing most commonly used governing parameters of synthetic jet, see e.g. [1,2]. Velocity scale is defined as time averaged blowing orifice centerline velocity over an entire cycle

$$
U_{0}=\frac{1}{T} \int_{0}^{T_{E}} u_{0}(r=0, t) \mathrm{d} t,
$$

where $T_{E}$ is blowing time and $T=1 / f$ is oscillation period. Alternatively, velocity scale can be defined as time and spatial averaged blowing orifice velocity

$$
\bar{U}=\frac{1}{T_{E}} \int_{0}^{T_{E}}\left(\frac{1}{A} \int_{0}^{D / 2} 2 \pi r u_{0}(r, t) \mathrm{d} r\right) \mathrm{d} t,
$$

where $D$ is orifice diameter and $A$ is orifice area. It is possible to show that two previously mentioned velocities are related as

$$
\bar{U}=2 U_{0} .
$$

a e-mail: tomas.hyhlik@fs.cvut.cz

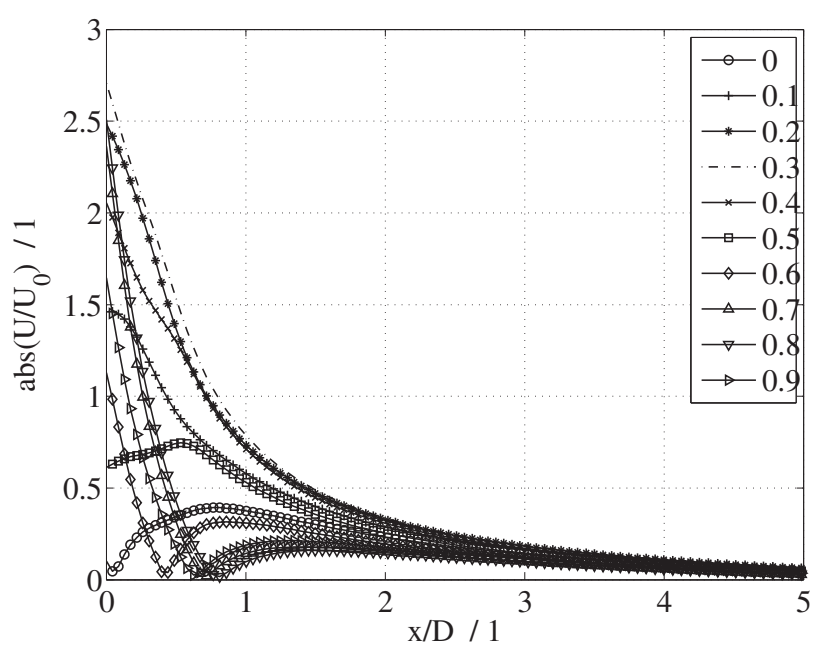

Fig. 1. Normalized phase averaged streamwise velocity magnitude at jet centerline at ten phases for $\mathrm{Re}=76$ and $\mathrm{S}=19.7$

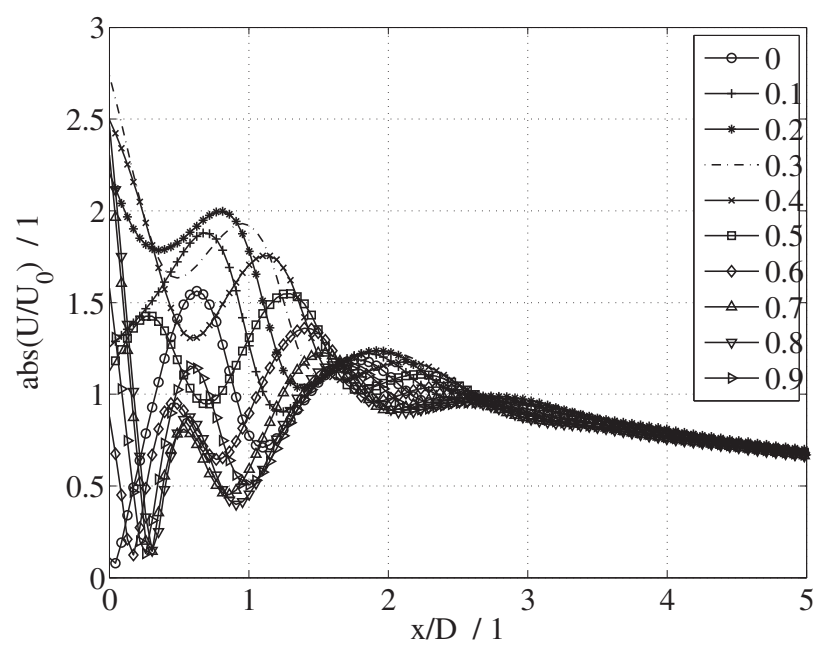

Fig. 2. Normalized phase averaged streamwise velocity magnitude at jet centerline at ten phases for $\mathrm{Re}=145$ and $\mathrm{S}=19.7$ 


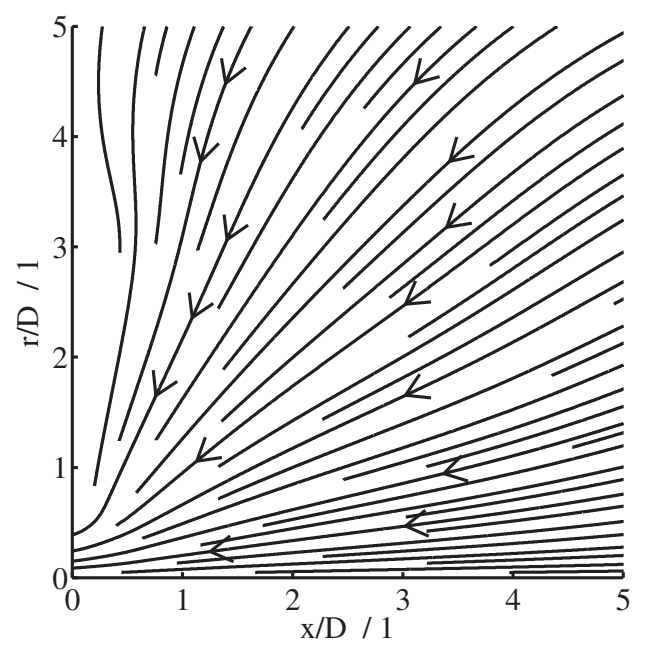

Fig. 3. Streamlines of first POD mode for $\mathrm{Re}=76$ and $\mathrm{S}=19.7$

Reynolds number can be defined using time averaged blowing orifice centerline velocity $U_{0}$ or using time and spatial averaged blowing orifice velocity $\bar{U}$ as

$$
R e=\frac{\bar{U} D}{v}=\frac{2 U_{0} D}{v} .
$$

Stokes number is defined as

$$
S=D \sqrt{\frac{2 \pi f}{v}},
$$

where $f$ is frequency. Stroke length of synthetic jet is length of fluid column that is pushed out during one cycle and can be calculated as

$$
L_{0}=U_{0} T \text {. }
$$

Dimensionless stroke length is defined as

$$
L=\frac{L_{0}}{D}=\frac{R e}{S^{2}} \pi
$$

\subsection{Existence of Synthetic Jet}

Criterion for synthetic jet formation is based on idea that vortex ring induced velocity has to overcome the suction velocity during ingestion stroke. According to reference [1] is ratio of vortex ring induced velocity to suction velocity proportional to $\mathrm{Re} / \mathrm{S}^{2}$. Criterion of synthetic jet existence is according to [1]

$$
\frac{R e}{S^{2}}>K
$$

where $K=0.16$ for axisymmetric synthetic jet. In the first studied case with Reynolds number $\mathrm{Re}=76$ and Stokes number $\mathrm{S}=19.7$ is $\mathrm{Re} / \mathrm{S}^{2}=0.1958$, in the second case with $\operatorname{Re}=145$ and $S=19.7$ is $\mathrm{Re} / \mathrm{S}^{2}=0.3736$ and in the third case with $\operatorname{Re}=329$ and $S=19.7$ is $\operatorname{Re} / S^{2}=0.8477$. Although criterion (8) in the first case is fulfilled, the topology of most energetically dominant POD mode depicted in the figure 3 together with figure 1 documented that it is the case where there is not synthetic jet. The two other cases

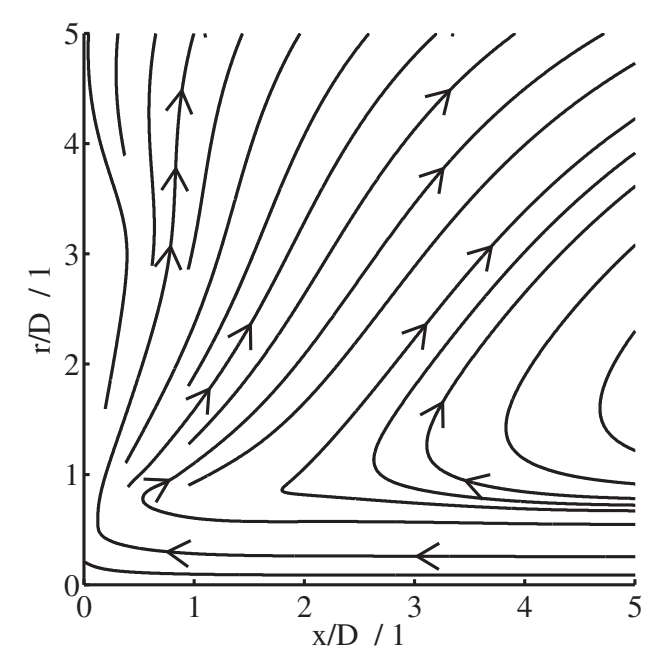

Fig. 4. Streamlines of first POD mode for $\mathrm{Re}=329$ and $\mathrm{S}=19.7$

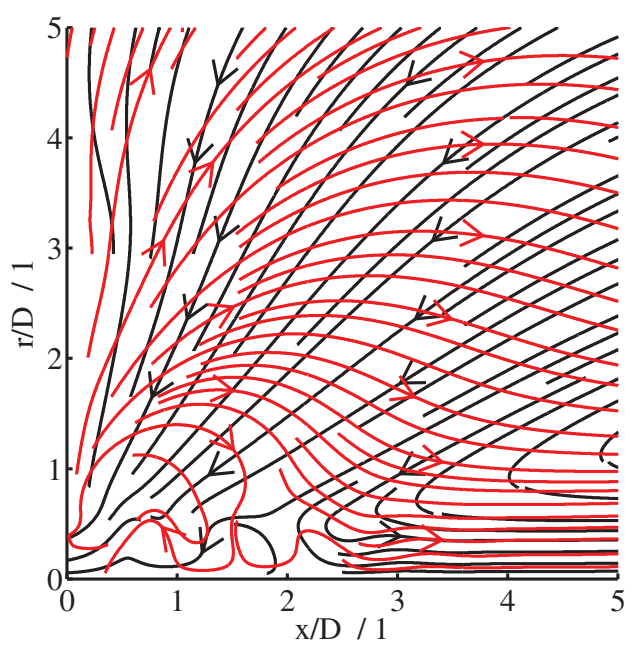

Fig. 5. Streamlines of second and third POD modes for $\operatorname{Re}=145$ and $\mathrm{S}=19.7$

are cases with synthetic jet creation as indicated in figures 2 and 4 . Criterion of synthetic jet existence according to [1] can be reformulated using dimensionless stroke length as

$$
L>\pi K
$$

Existence of synthetic jet is extensively studied in the literature experimentally, numerically and theoretically. As an example of experimental study can be mentioned reference [2]. There is also included extensive literature review in the mentioned reference.

\section{Numerical simulation}

Unsteady incompressible laminar flow simulation has been performed using commercial solver ANSYS Fluent. The flow is assumed to be axisymmetric and is simulated on computational mesh with 81550 cells by using non iterative time advancement method with second order implicit scheme. Fractional step scheme is used for pressure velocity coupling. Convective terms are discretized using third 


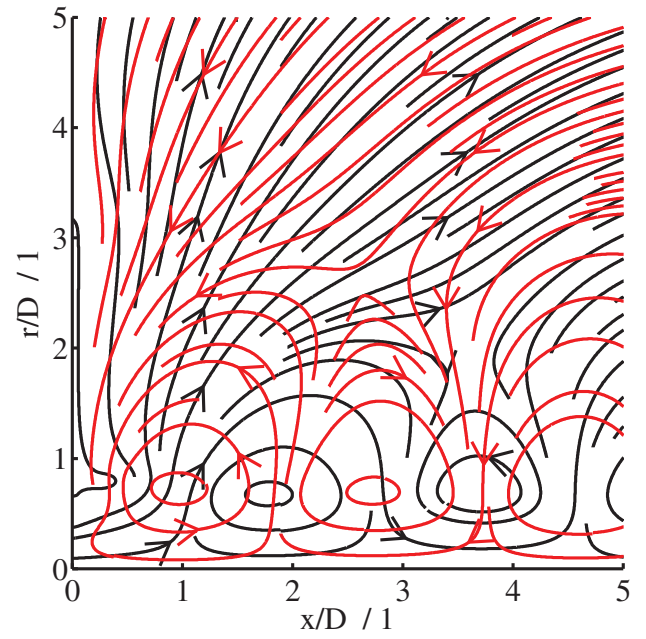

Fig. 6. Streamlines of second and third POD modes for $\mathrm{Re}=329$ and $\mathrm{S}=19.7$

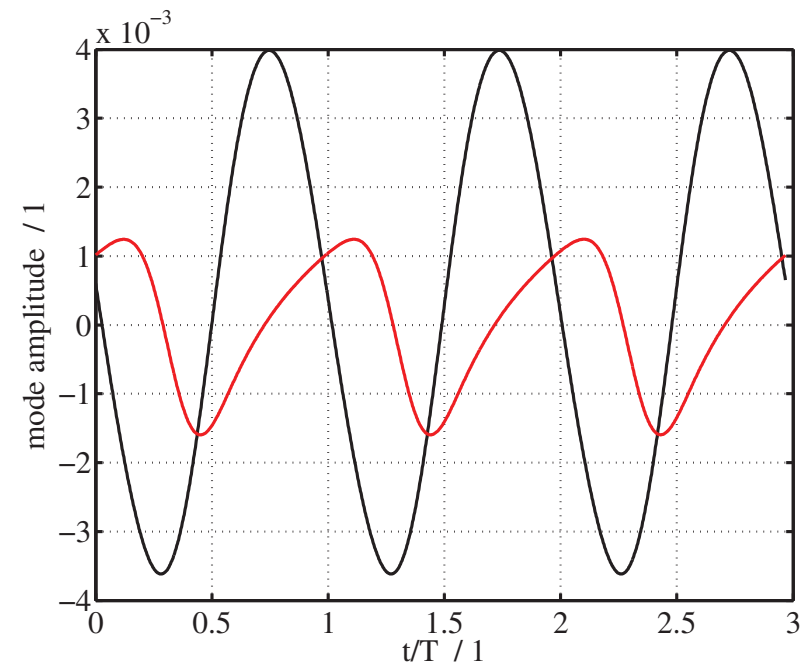

Fig. 7. Temporal amplitudes of second and third POD modes for $\operatorname{Re}=145$ and $S=19.7$

order MUSCL scheme. Both the flow in the orifice and actuator cavity is included in the simulation to get more accurate results. The effect of oscillating diaphragm on the left is replaced by velocity boundary condition which should guarantee achieving of required Reynolds number. The equation for inlet velocity is then

$$
u(t)=\frac{\pi}{2} \frac{\operatorname{Re\eta }}{\rho D} \frac{D^{2}}{D_{L}^{2}} \sin (2 \pi f t),
$$

where $\eta$ is dynamic viscosity, $\rho$ is air density and $D_{L}$ is inlet diameter i.e. diameter of oscillating diaphragm.

\section{Modal decomposition}

Modal decomposition is based on set of flow fields from numerical simulation which are equidistantly sampled with time step $\Delta t$

$$
\mathbf{U}=\left[\mathbf{u}_{1} \ldots \mathbf{u}_{\mathbf{N}-\mathbf{1}} \mathbf{u}_{\mathbf{N}}\right]
$$

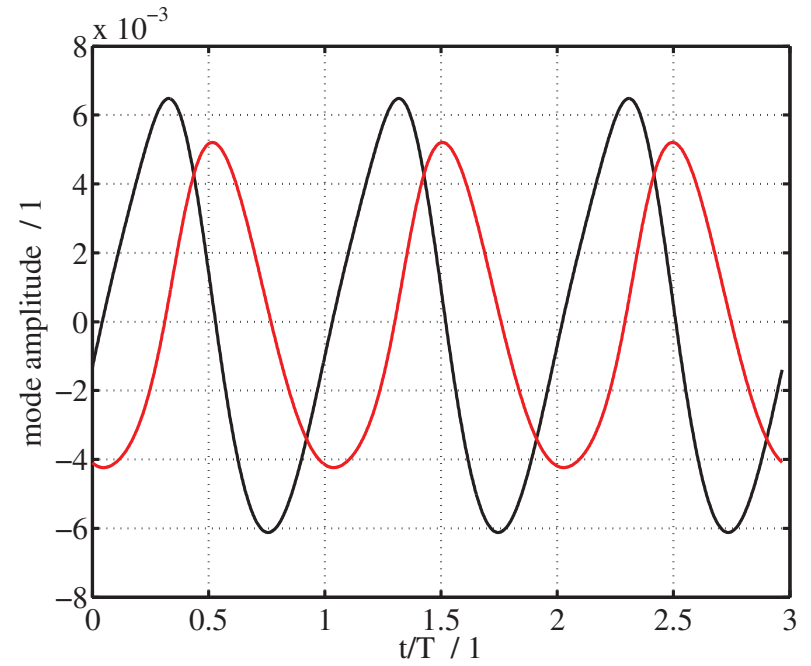

Fig. 8. Temporal amplitudes of second and third POD modes for $\operatorname{Re}=329$ and $\mathrm{S}=19.7$

Proper orthogonal decomposition (POD) method is commonly used modal decomposition technique. The main objective of POD is to obtain an optimal low dimensional basis for representing of high dimensional simulation data [3, 4]. The method is capable of extracting information from snapshots of the flow field. POD decomposes a given flow field into an orthonormal system of spatial modes $\mathbf{X}$ and corresponding mode amplitudes $\mathbf{T}$

$$
\mathbf{U}=\mathbf{X} \mathbf{T} \text {. }
$$

This method determines the most energetic structures by diagonalizing the correlation matrix computed from the snapshots

$$
\mathbf{C}=\mathbf{U}^{T} \mathbf{W U}
$$

where $\mathbf{W}$ is weighting matrix with cell volumes on diagonal. The POD modes are based on the solution of eigenvalue problem [3]

$$
\mathbf{C v}_{\mathbf{i}}=\lambda_{i} \mathbf{v}_{\mathbf{i}}, \quad i=1, \ldots, N .
$$

Eigenvalues $\lambda_{i}$ are proportional to the energy of given mode. Temporal amplitude $\mathbf{T}$ can be obtained by rescaling of orthonormal eigenvector $\mathbf{v}_{\mathbf{i}}$ and spatial modes $\mathbf{X}$ are evaluated using inversion from equation (12) [3].

All of studied regimes of synthetic jet flow are analyzed using POD method. Results of POD decomposition are based on the data from last three periods of CFD computation, where nine hundreds of snapshots per one period is used.

\section{Results}

Analyzed data are normalized by using averaged blowing orifice centerline velocity over an entire cycle $U_{0}$ for every case. Normalized phase averaged streamwise velocity at jet centerline depicted in figure 1 shows together with streamlines of the first POD mode in the figure 3 the case where jet is not synthetized. Temporal amplitude corresponding with first spatial mode in the figure 3 is harmonic function with average equal to zero, it means that in this case 
is fluid sucked in from the whole region outside the orifice and blown out to whole orifice surroundings. Contrary to the first case the second case shows different behavior, i.e. there is a suction along the wall in the radial direction and blowing is from the wall in the axial direction as shown in the figure 4. It is necessary to mention that temporal amplitude corresponding to first POD mode depicted in the figure 4 is almost constant negative function and therefore it flows in the opposite direction then is drawn.

Streamlines of second and third mode depicted in the figures 5 and 6 indicate vortex creation, especially the structure shown in the figure 6 is typical vortex street like structure [4]. Corresponding temporal amplitudes are shown in figures 7 and 8 . There is a phase shift between second and third temporal amplitude which also indicates creation of vortex street. Vortex like structure in the figures 5 and 7 is significantly weaker and is vanishing close to orifice unlike the case in the figures 6 and 8. Contours of streamwise velocity component of third POD mode are shown in the figure 9 for the case of $\mathrm{Re}=329$ and $\mathrm{S}=19.7$. The structure of the fifth POD mode depicted in the figure 10 is similar to the structure of second and third mode, but it is finer. Similar conclusion is connected with temporal amplitudes of fourth and fifth mode shown in the figure 11, where is two times higher frequency than in the figure 8. It has been observed that POD modes number six and seven are connected with three times higher frequency and more finer spatial structure and similar behaviour is observed for higher POD modes with decreasing amount of energy included in the modes.

\section{Conclusions}

Modal decomposition of synthetic jet flow regimes has been done by using POD method. The POD modes reveal the structure of flow fields. It has been shown that typical vortex street like structure of POD modes can be observed in the case of $\operatorname{Re}=329$ and $S=19.7$. The case with $\operatorname{Re}=145$ and $S=19.7$ exhibits evidence of weak vortex structure close to orifice and weak synthetic jet. The case with $\operatorname{Re}=76$ and $S=19.7$ shows behavior without synthetic jet creation.

\section{Acknowledgement}

The support from Czech Science Foundation under Grant No. 14-08888S is gratefully acknowledged.

\section{References}

1. R. Holman, Y. Utturkar, R. Mittal, B. L. Smith, L. Cattafesta, AIAA Journal, 43(10), (2005), 2110-2116

2. Z. Trávníček, Z. Broučková, J. Kordík, AIAA Journal, 50(9), (2012), 2012-2017

3. O. Frederich, D. M. Luchtenburg, Seventh International Symposium on Turbulence and Shear Flow Phenomena, (Ottawa 2011)

4. T. Hyhlík, P. Železný, J. Čížek, EPJ Web of Conferences, 45, (2013), 01043

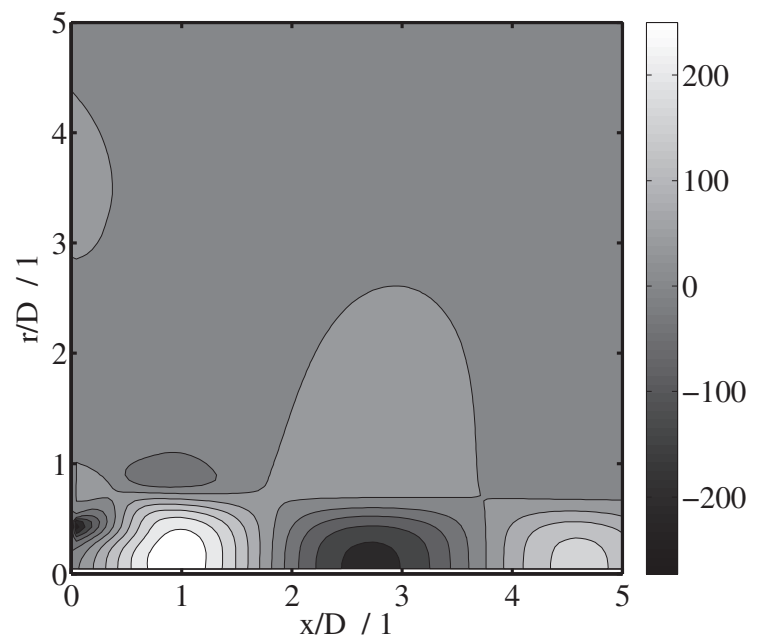

Fig. 9. Contours of streamwise velocity component of third POD mode for $\mathrm{Re}=329$ and $\mathrm{S}=19.7$

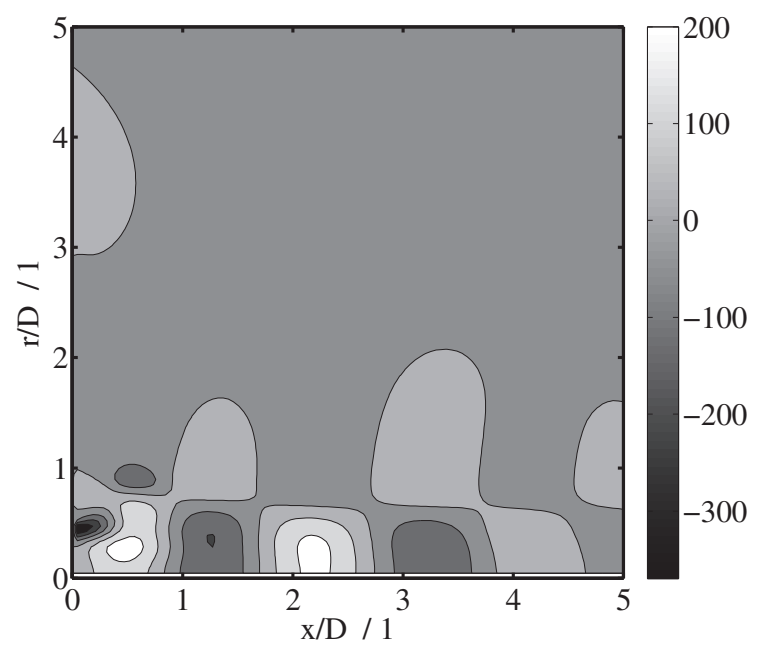

Fig. 10. Contours of streamwise velocity component of fourth POD mode for $\mathrm{Re}=329$ and $\mathrm{S}=19.7$

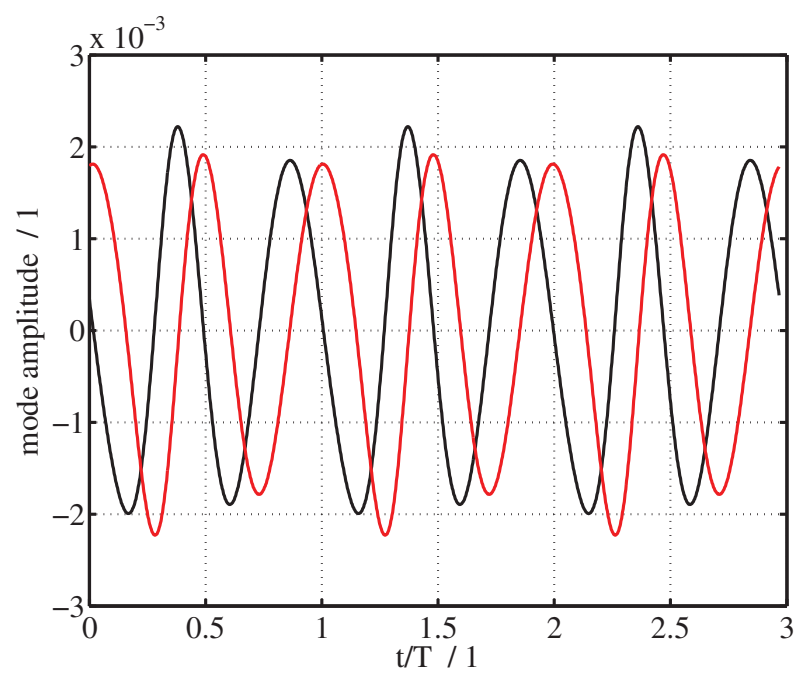

Fig. 11. Temporal amplitudes of fourth and fifth POD modes for $\mathrm{Re}=329$ and $\mathrm{S}=19.7$ 